\title{
Microsolvation of Glycine: A DFT Study
}

Steven M. Bachrach

Department of Chemistry, Trinity University, 1 Trinity Place, San Antonio, TX 78212

sbachrach@trinity.edu

Supporting Information Available: Complete citation of Reference 27, Tables S1 and S2, relative energies and drawings of all structures computed at B3L YP/6-31G(d) and coordinates, energies and drawings (part 2) of all structures computed at PBE1PBE/6$311+\mathrm{G}(\mathrm{d}, \mathrm{p})$.

PART 2 
Figure S5. PBE1PBE/6-311+G(d,p) optimized structures of $\mathbf{N 4}$ and $\mathbf{Z 4}$.

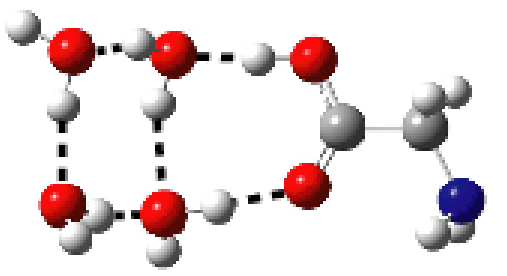

N4-a

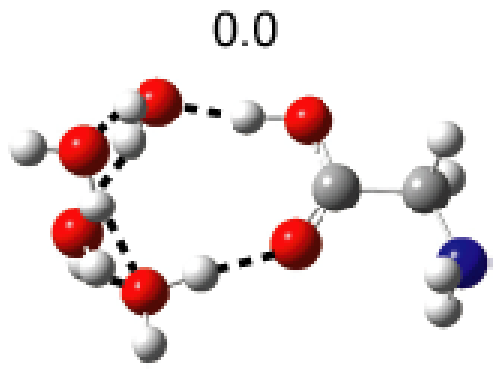

N4-d

1.08

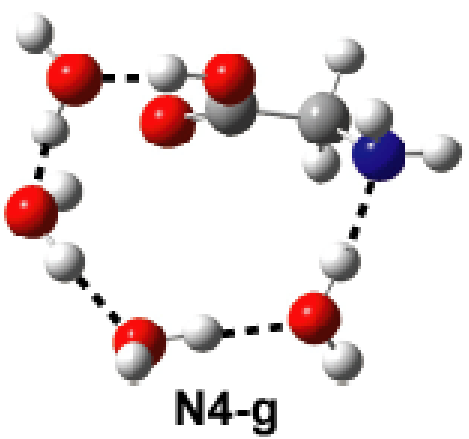

1.84

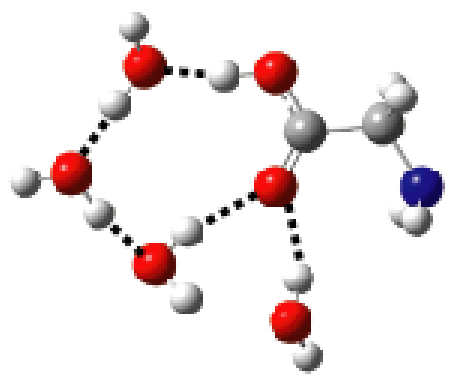

N4-j

3.48

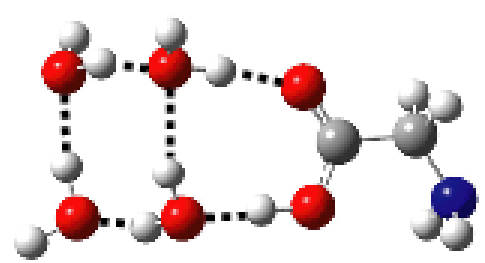

N4-b

0.72

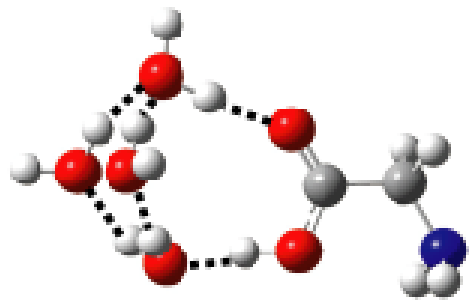

N4-e

1.70

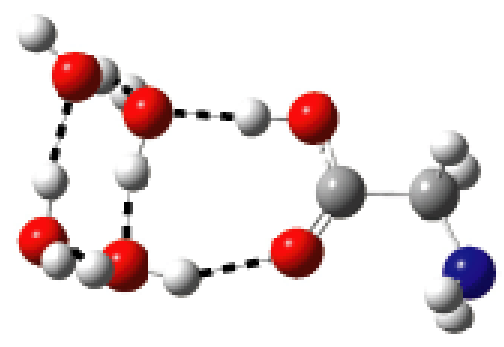

N4-h

2.04

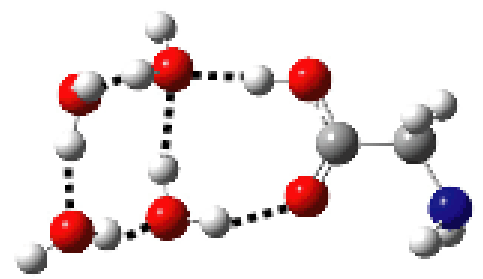

N4-C

0.93

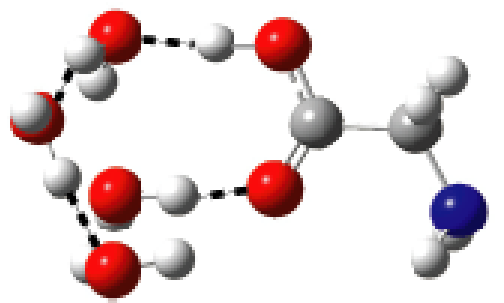

N4-f

1.73

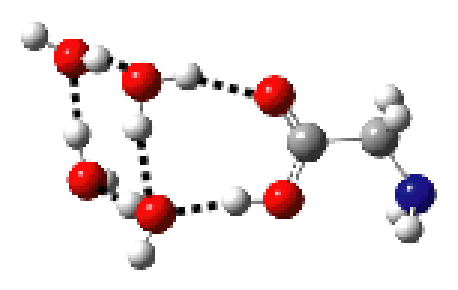

N4-i

2.27 
Figure S5 (continued).

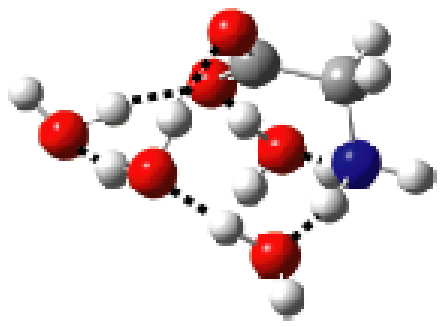

Z4-b

6.53

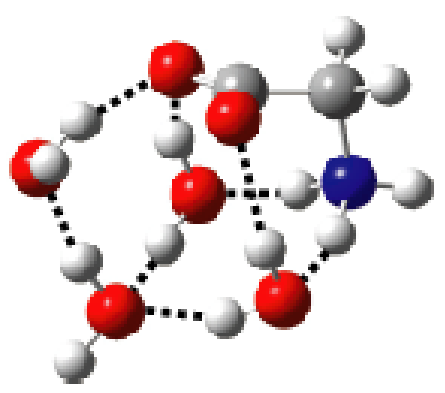

Z4-e

7.63

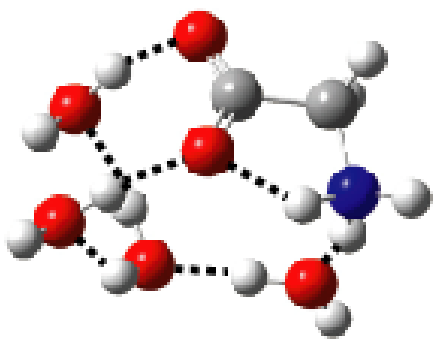

Z4-C

6.69

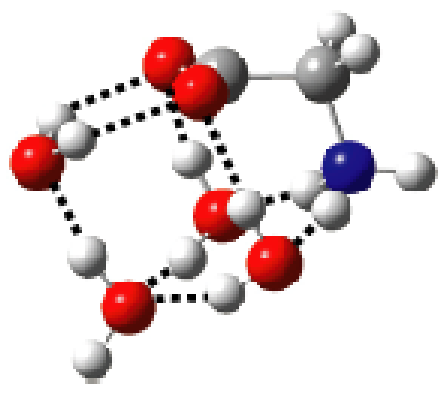

Z4-f

7.77

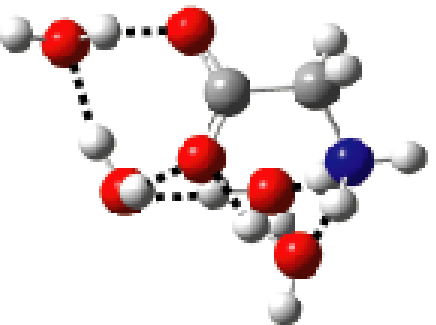

Z4-a

6.04

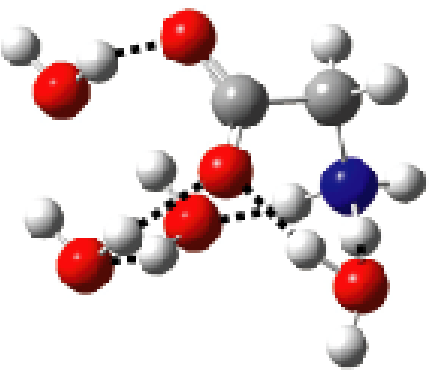

Z4-d

7.39

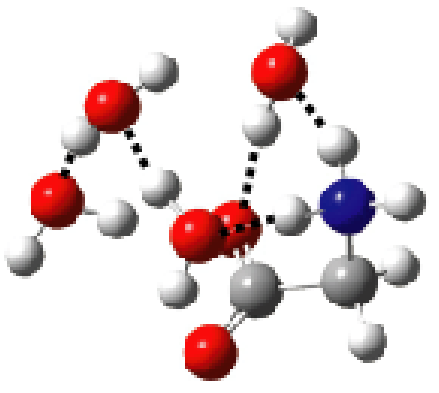

Z4-g

9.53 
Figure S6. PBE1PBE/6-311+G(d,p) optimized structures of N5 and Z5.

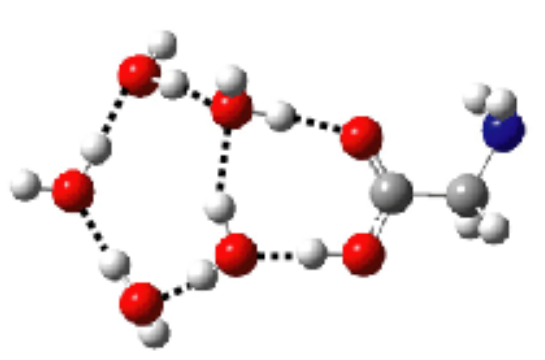

N5-a

0.0

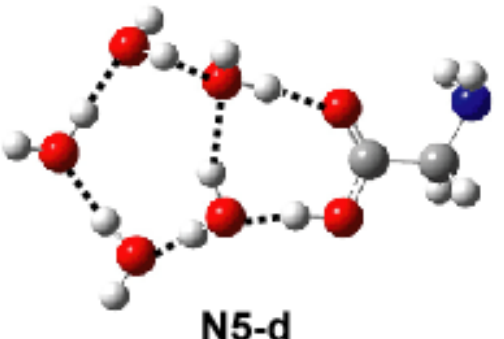

0.25

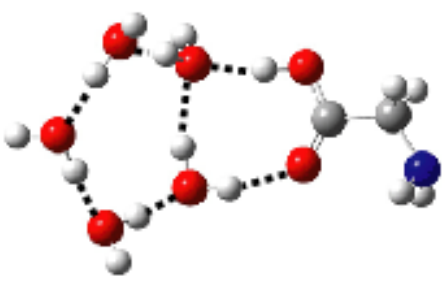

N5-g

0.72

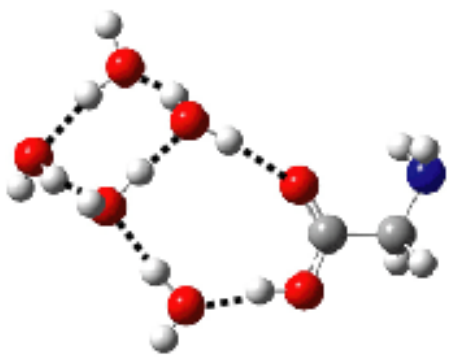

N5-j

1.79

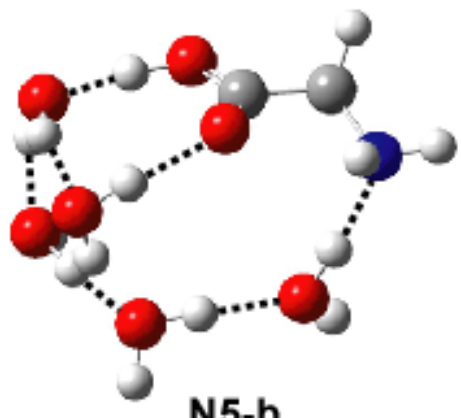

N5-b

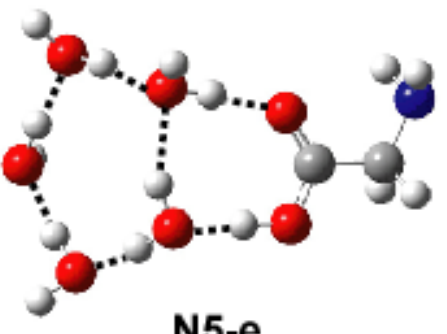

N5-e

0.27

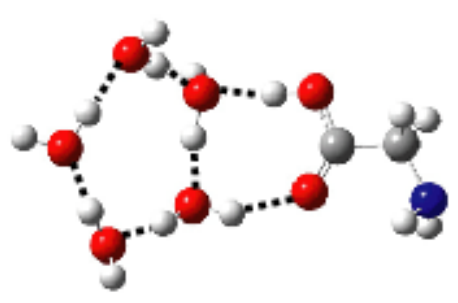

N5-h

1.28

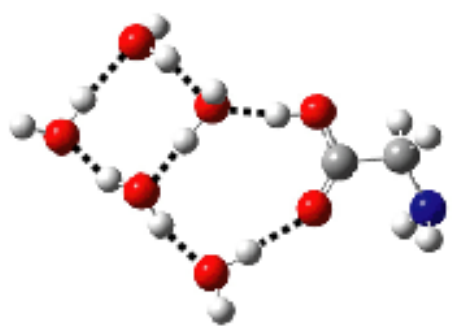

N5-k

1.88

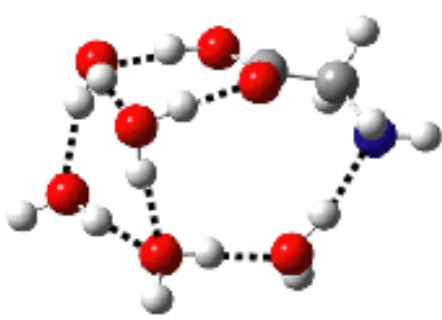

N5-c

0.17

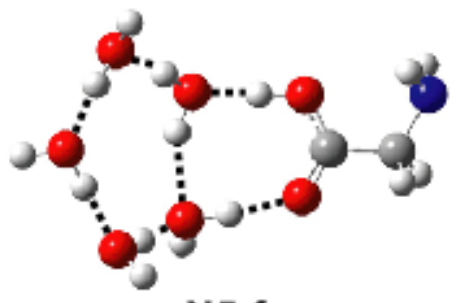

N5-f

0.68

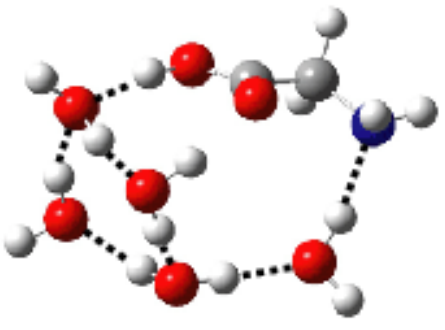

N5-i

1.55

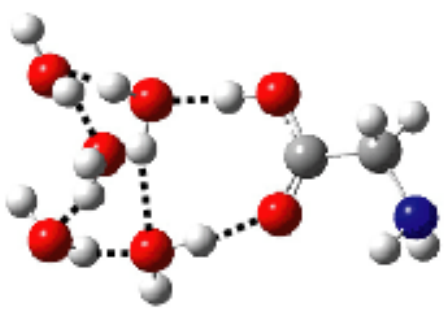

N5-I

2.27 
Figure S6. (continued)

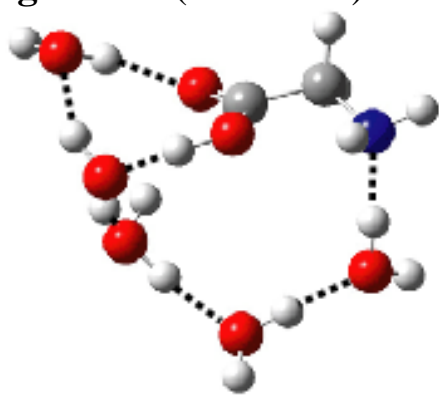

N5-m

2.33

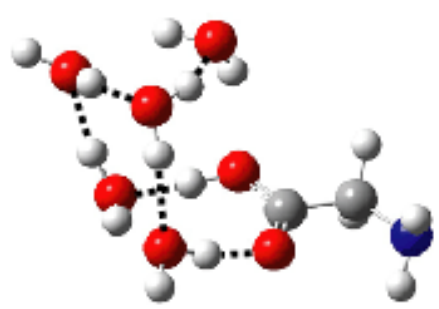

N5-p

3.03

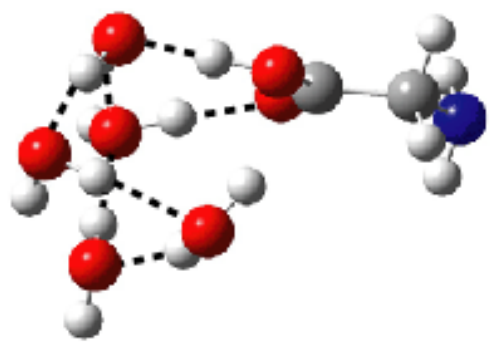

N5-s

3.60

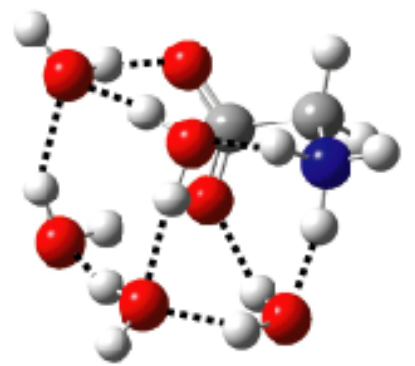

Z5-a

2.68

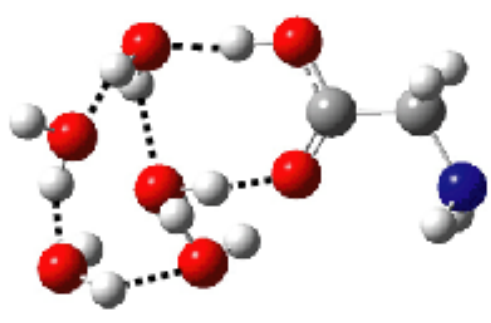

N5-n

2.55

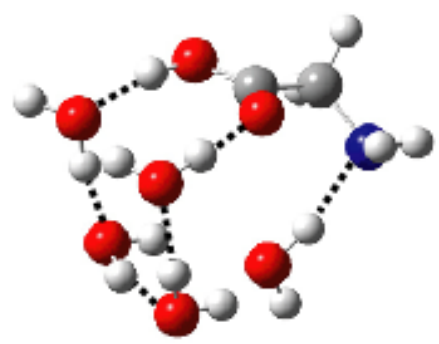

N5-q

3.05

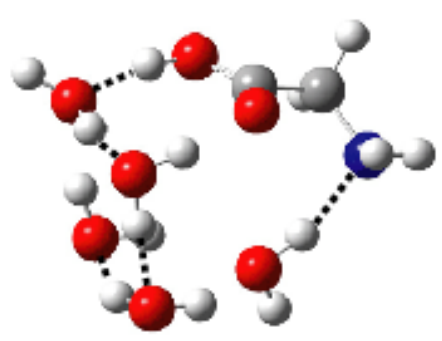

N5-O

2.63

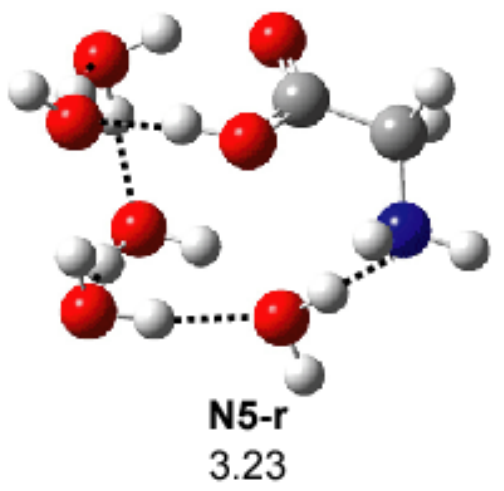


Figure S6. (continued)

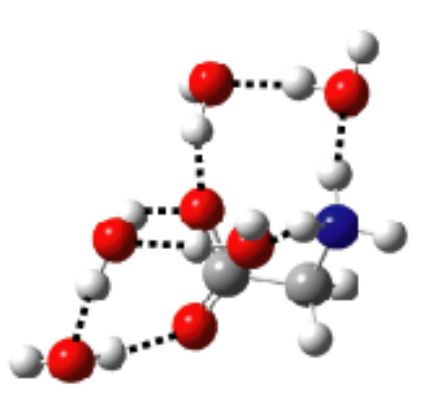

Z5-d

4.54

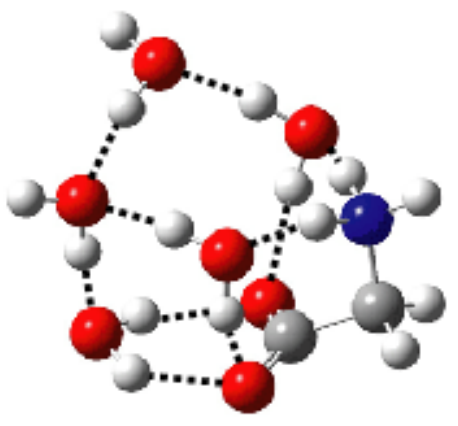

Z5-g

5.41

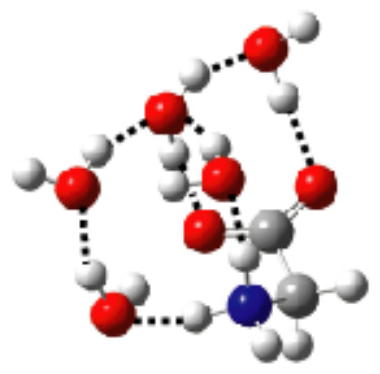

Z5-j

6.44

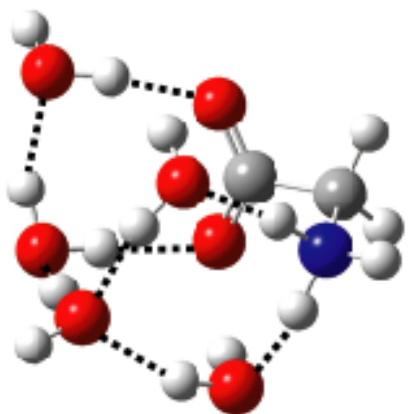

Z5-e

4.58

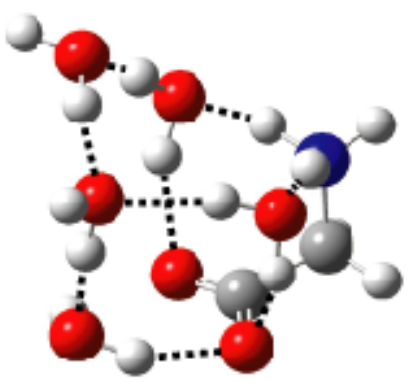

Z5-h

5.53

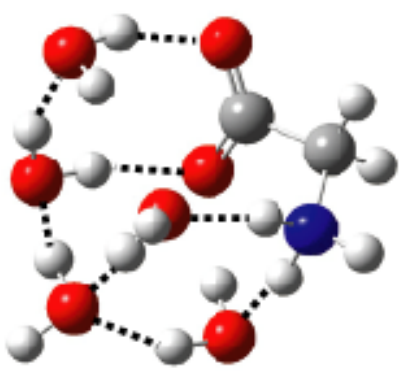

Z5-k

8.65

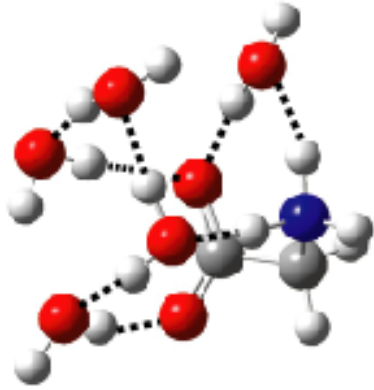

Z5-f

5.15

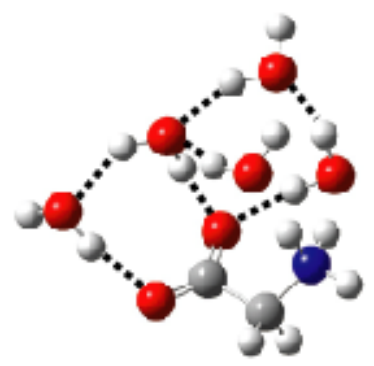

Z5-i

6.26 
Figure S7. PBE1PBE/6-311+G(d,p) optimized structures of N6 and Z6 


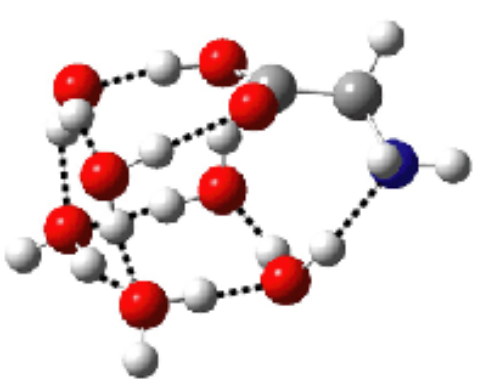

N6-a

0.0

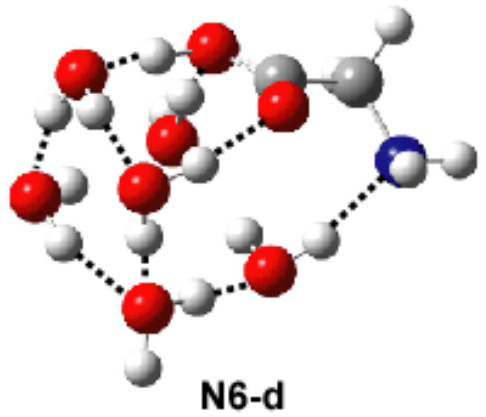

0.86
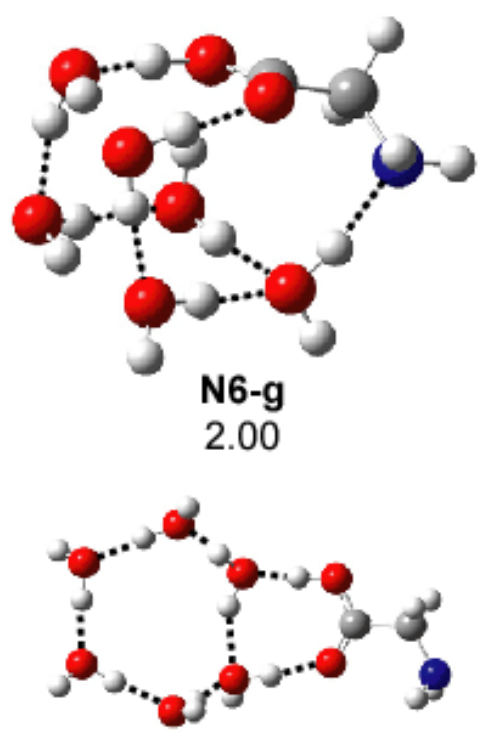

N6-j

2.40

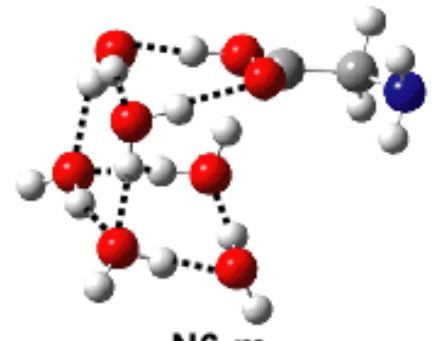

N6-m

3.34

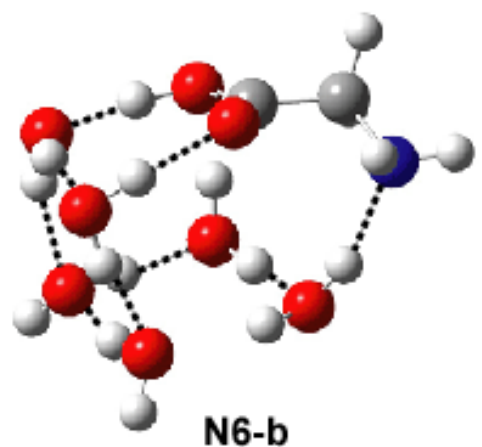

0.09

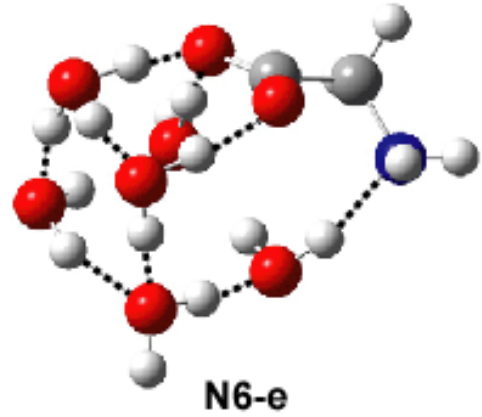

1.17

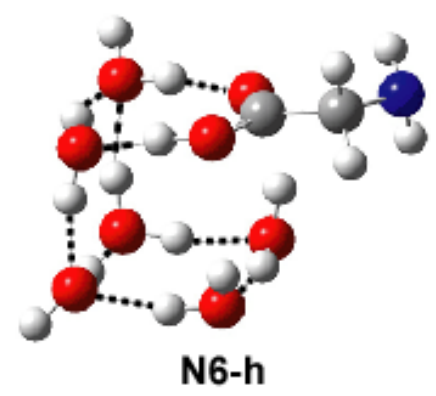

2.06

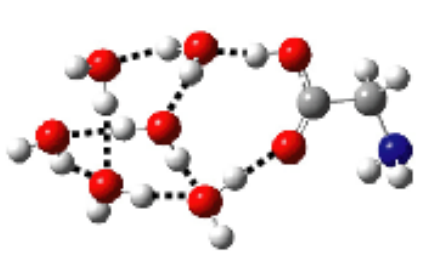

N6-k

2.53

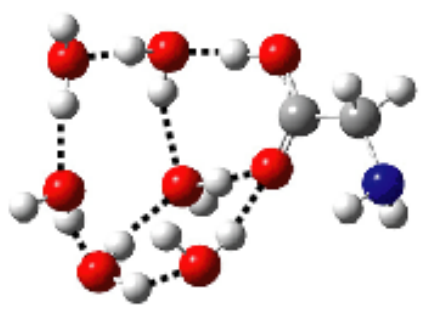

N6-n

3.45

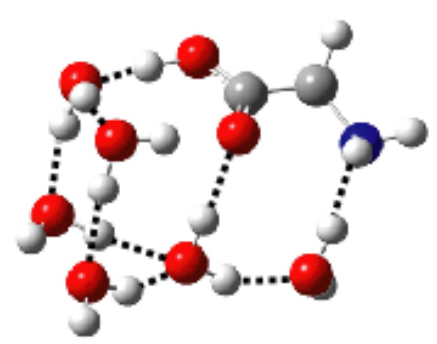

N6-C

0.54

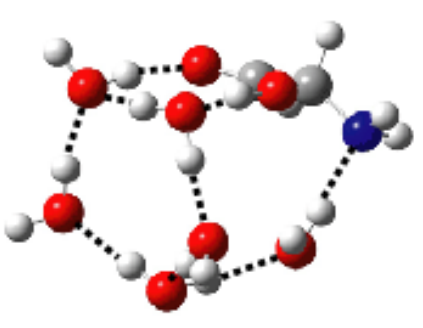

N6-f

1.98

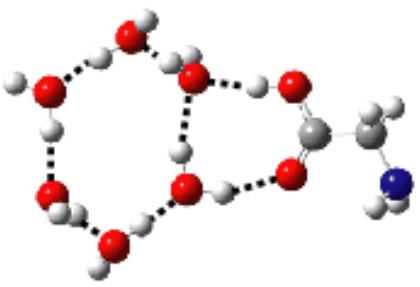

N6-i

2.24

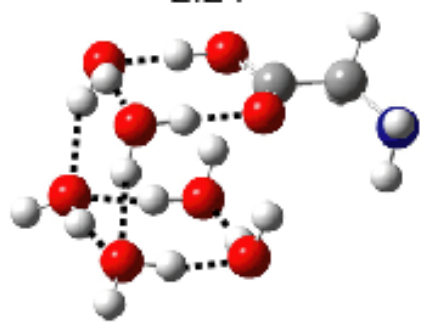

N6-I

3.14

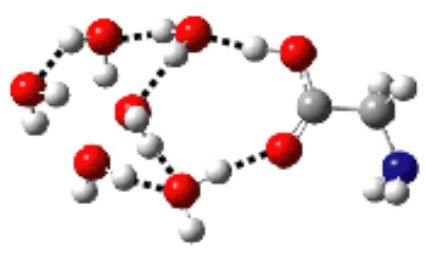

N6-o

3.57

Figure S7. (continued) 


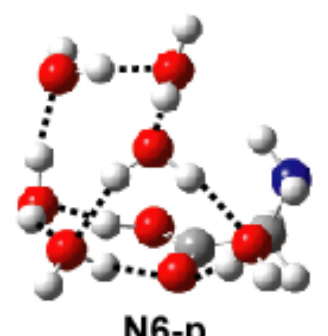

N6-p

4.35

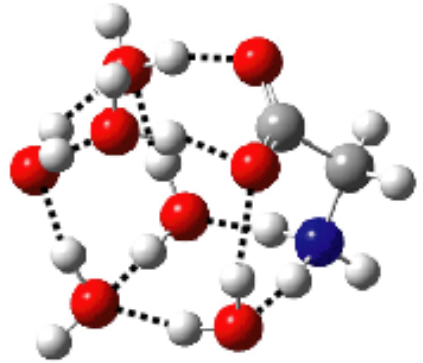

Z6-a

1.68

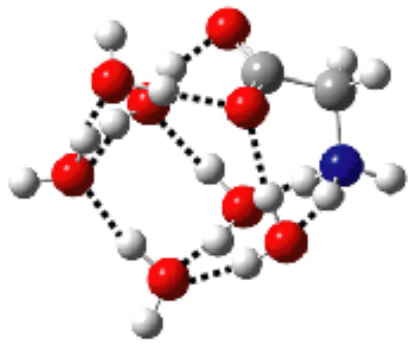

Z6-d

2.88

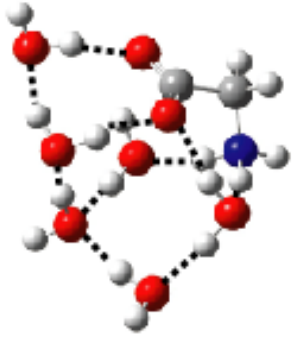

Z6-g

3.28

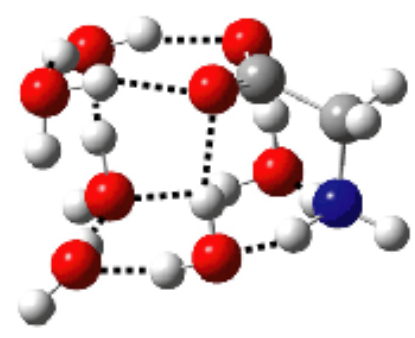

Z6-j

5.27

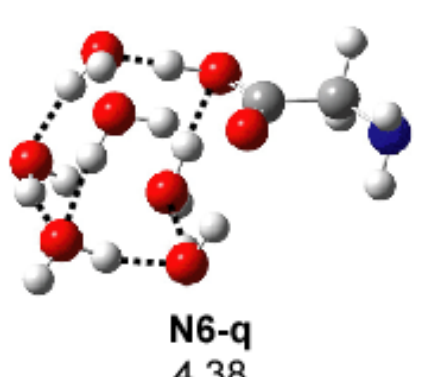

4.38

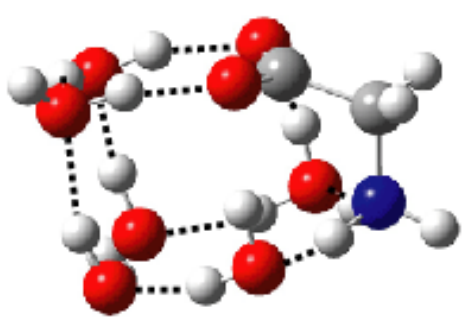

Z6-b

2.02

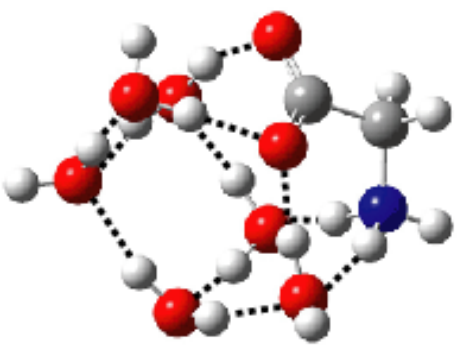

Z6-e

2.92

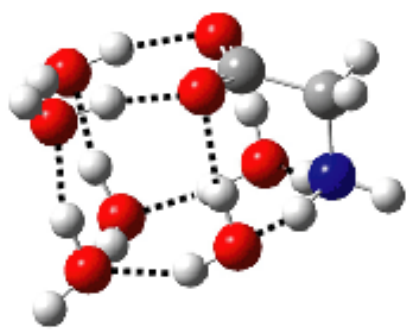

Z6-h

3.94

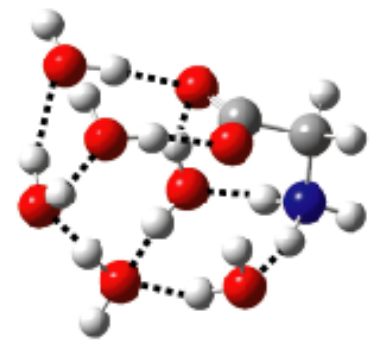

Z6-k

5.46

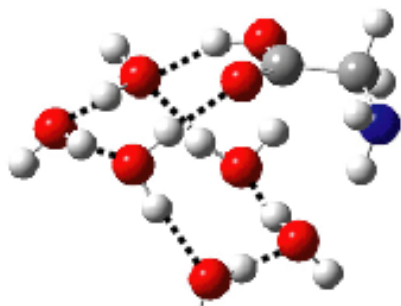

N6-r

5.81

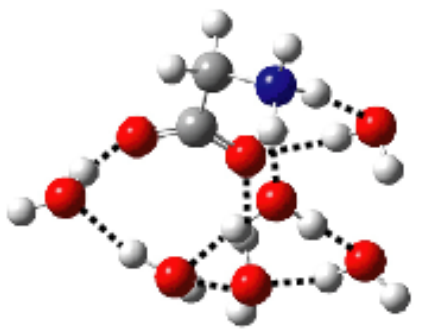

Z6-C

2.46

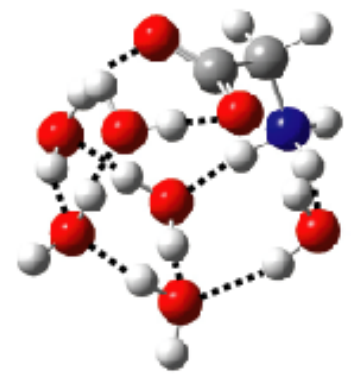

Z6-f

2.99

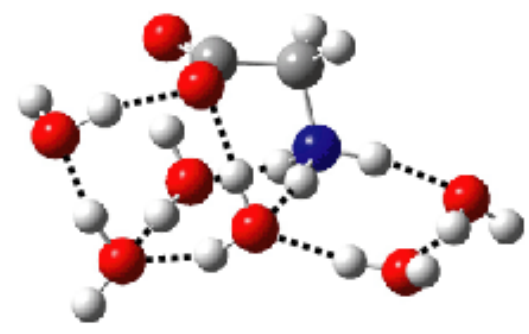

Z6-i

5.23 
Figure S8. PBE1PBE/6-311+G(d,p) optimized structures of N7 and Z7.

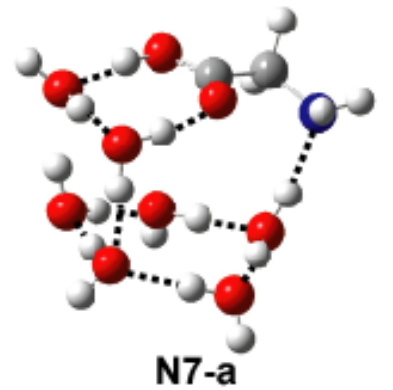

0.01

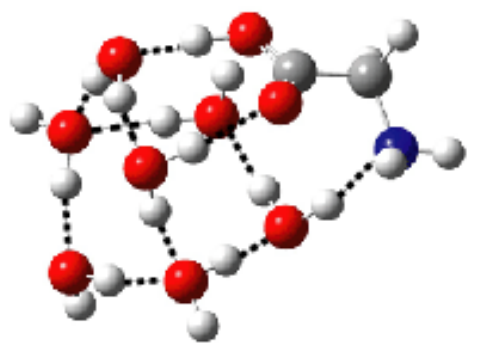

N7-d

0.83

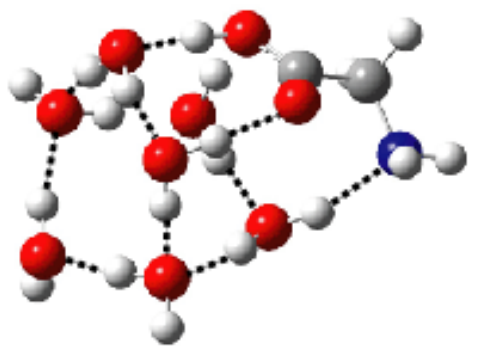

N7-g

1.22

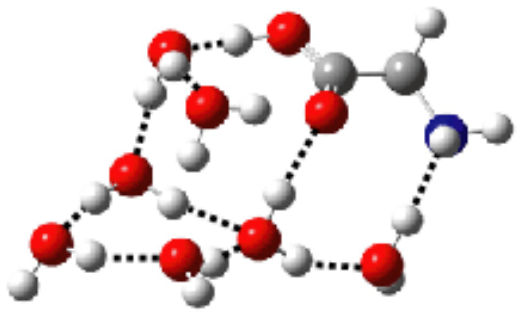

N7-j

1.40

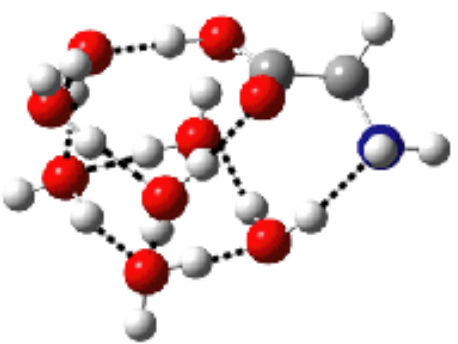

N7-b

0.39

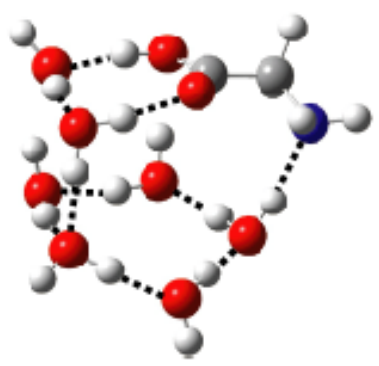

N7-e

0.95

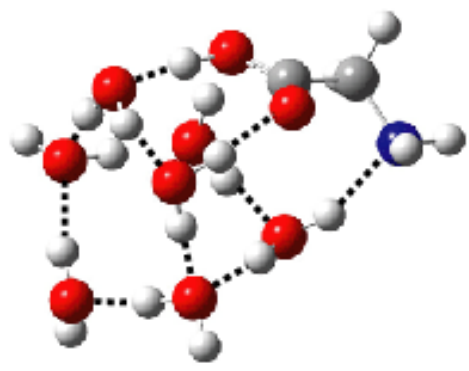

N7-h

1.31

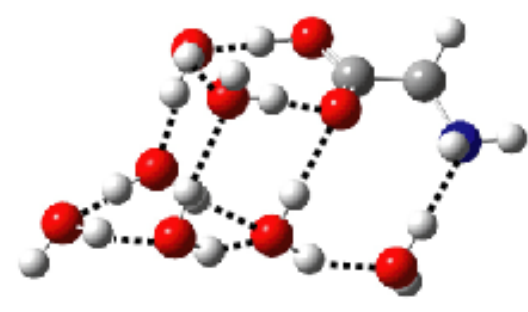

N7-k

1.68

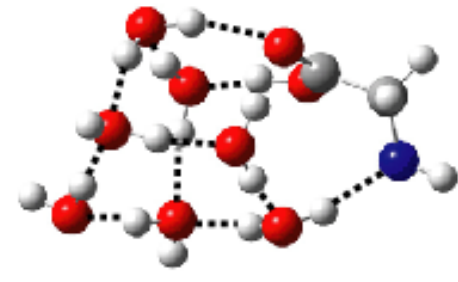

N7-c

0.57

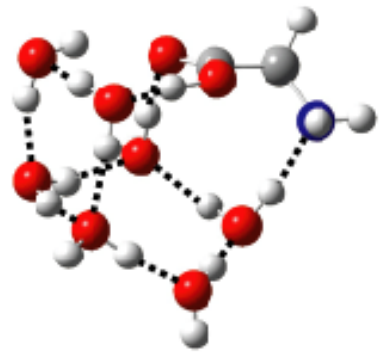

N7-f

1.19

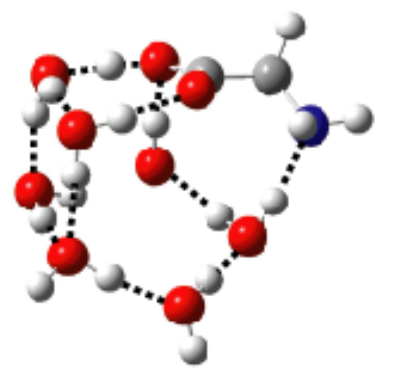

N7-i

1.40

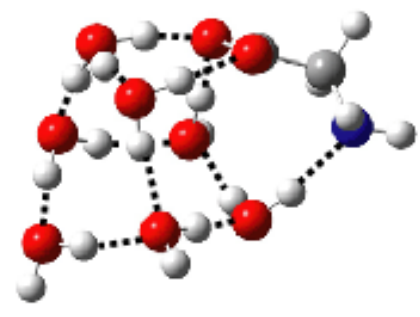

N7-I

1.99 
Figure S8. (continued)

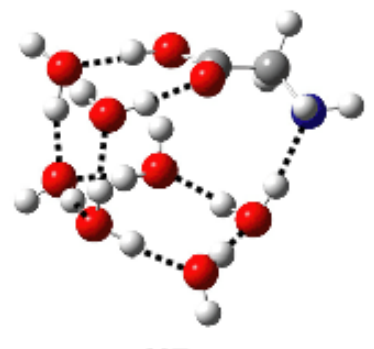

N7-m

2.38

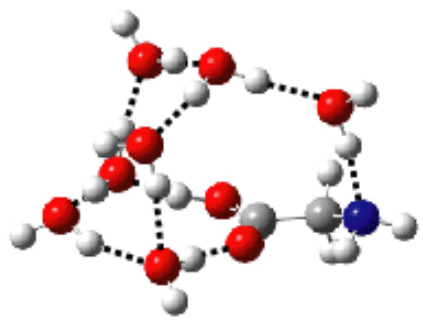

N7-p

2.77

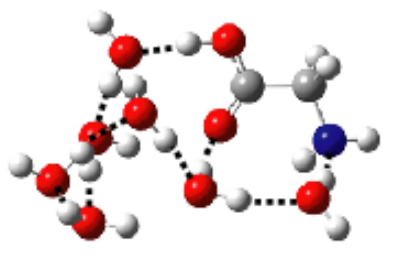

N7-s

3.23

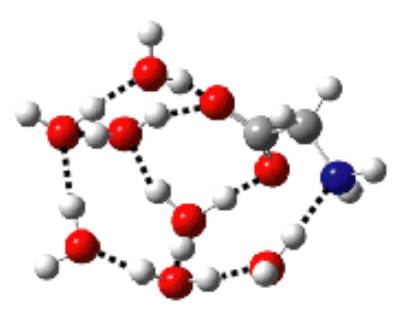

N7-n

2.61

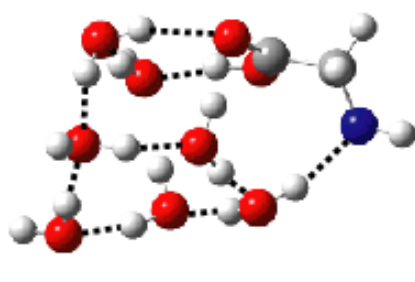

N7-q

2.78

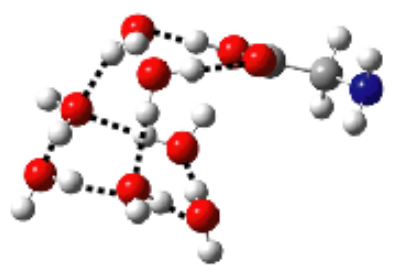

N7-t

3.47

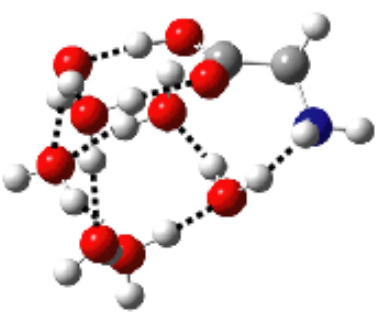

N7-O

2.67

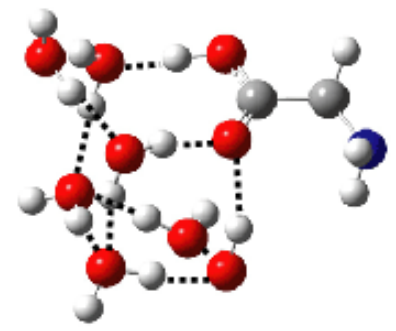

N7-r

2.90

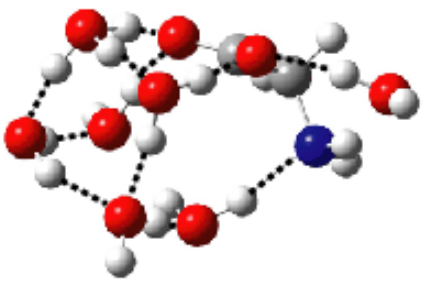

N7-u

4.24 
Figure S8. (continued)

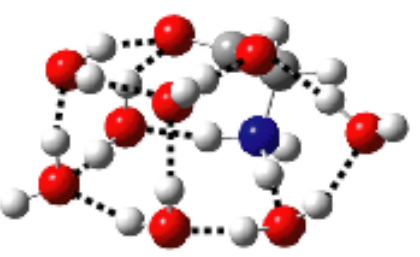

Z7-a

0.0

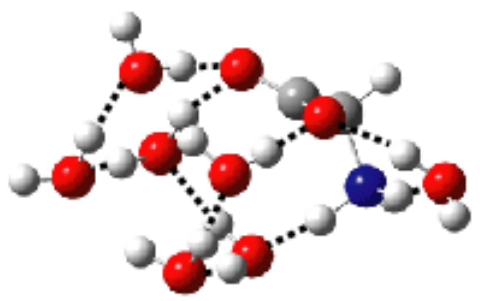

Z7-d

1.51

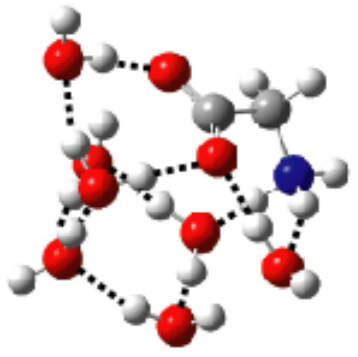

Z7-g

2.19

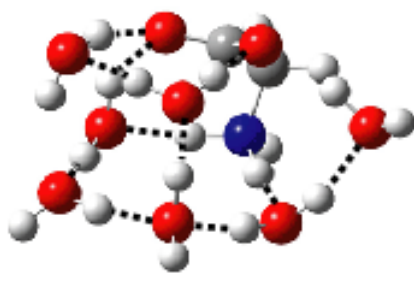

Z7-j

2.43

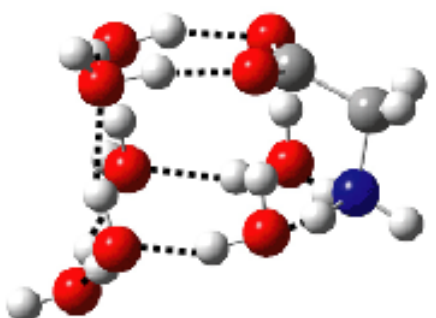

Z7-m

3.42

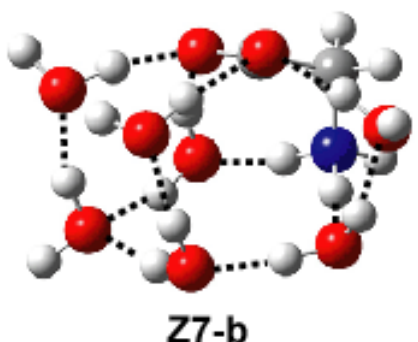

1.19

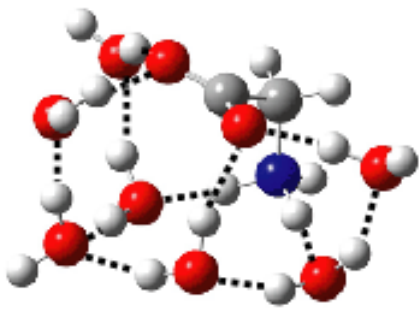

Z7-e

1.58

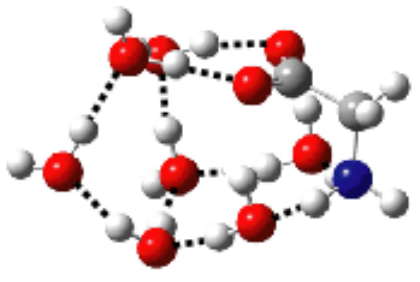

Z7-h

2.32

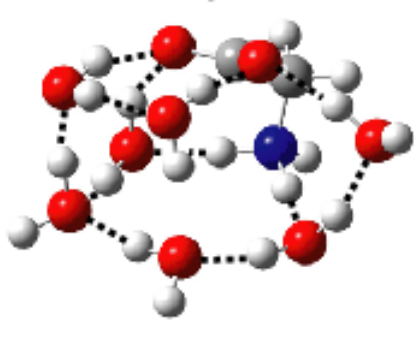

Z7-k

2.94

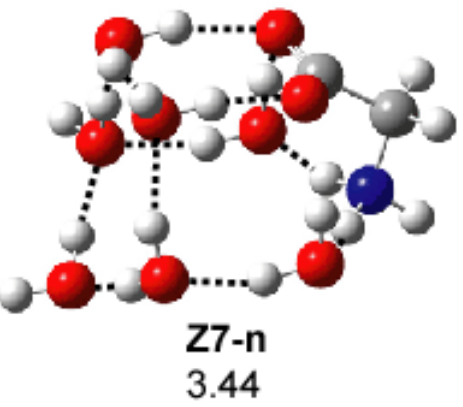

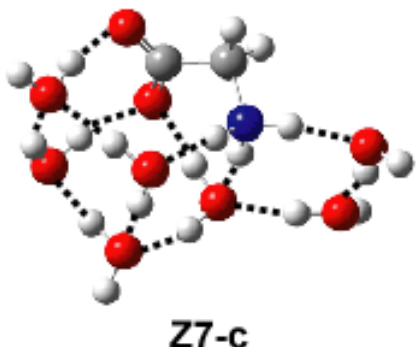

1.40

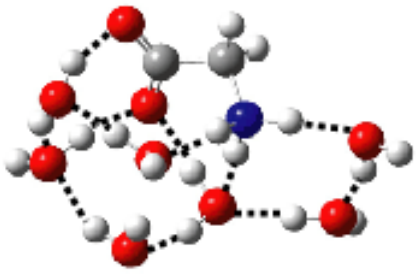

Z7-f

2.15

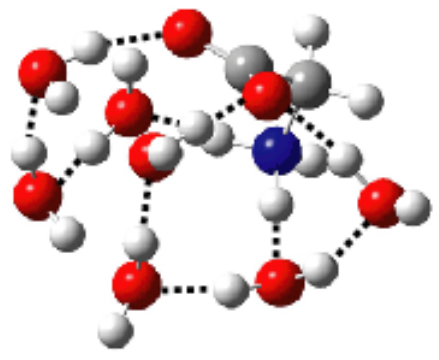

Z7-i

2.41

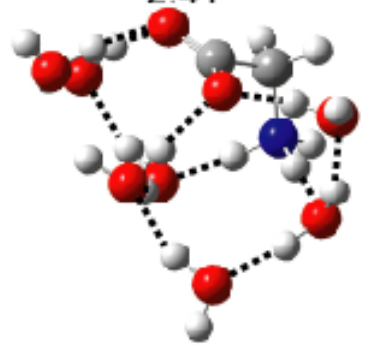

Z7-I

3.12

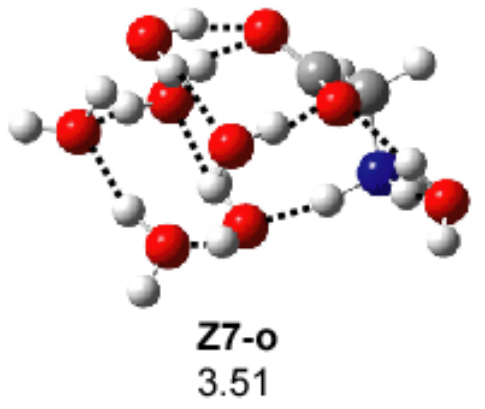


Figure S8. (continued)
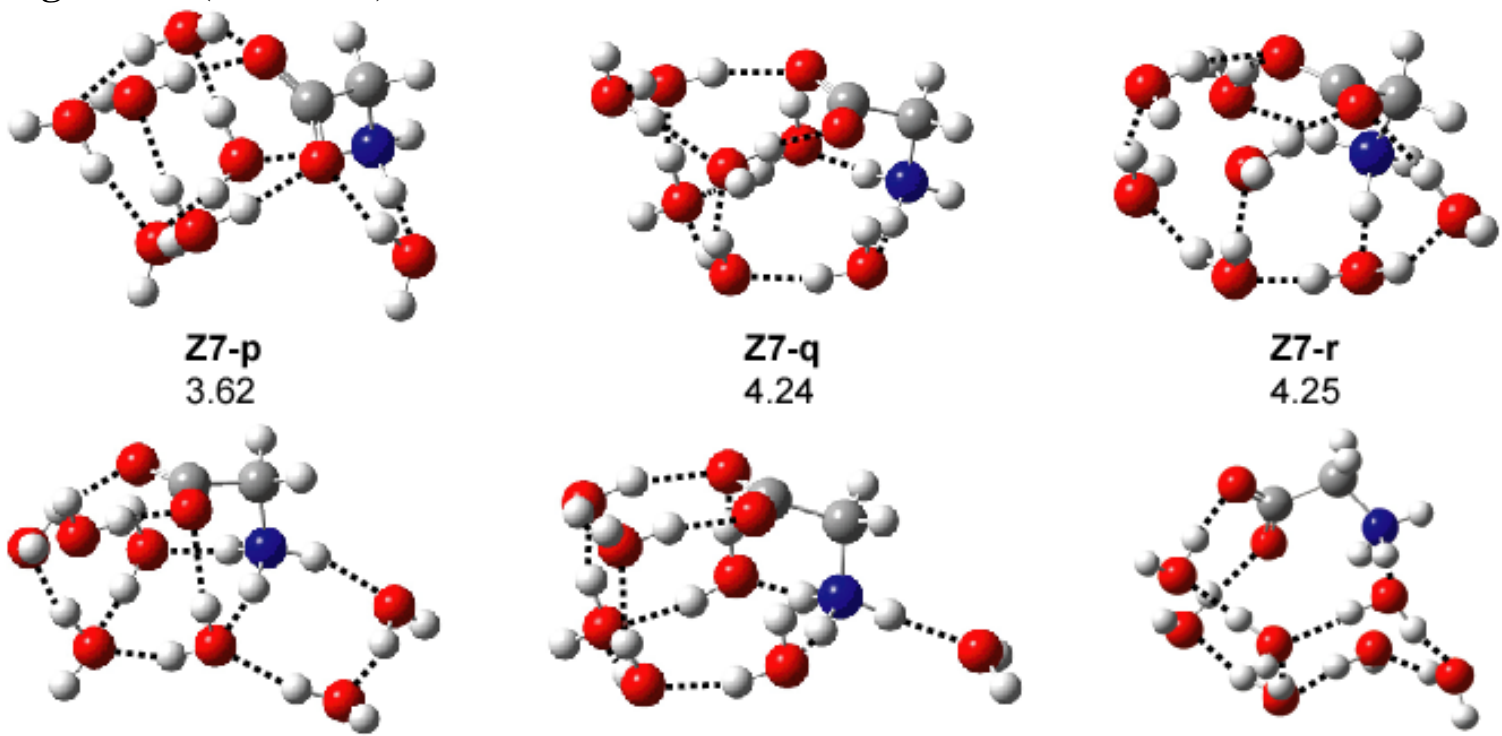

Z7-s

4.30
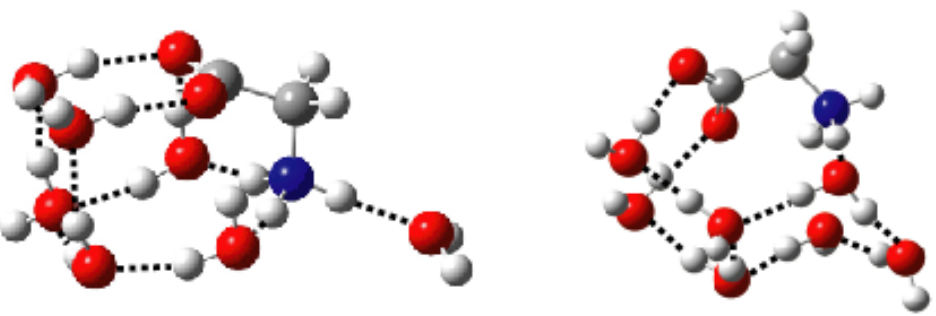

Z7-t

4.34

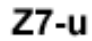

4.64
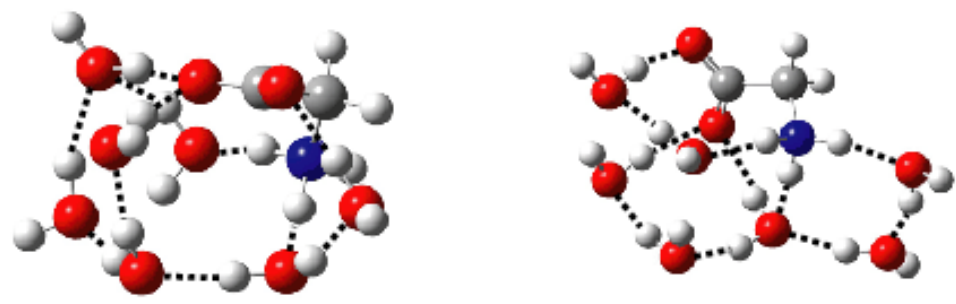

Z7-v

Z7-w

5.08

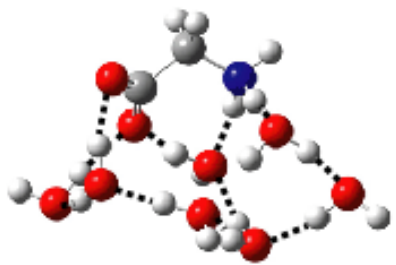

4.85

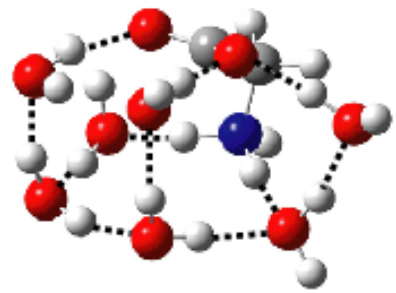

5.34

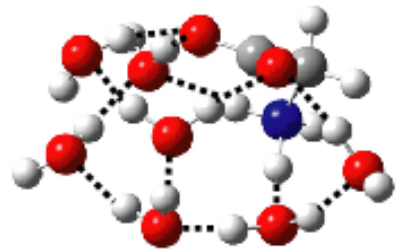

Z7-y

Z7-z

6.28

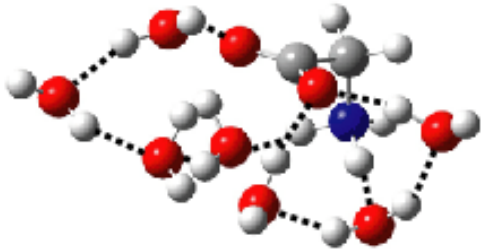

Z7-aa

6.29

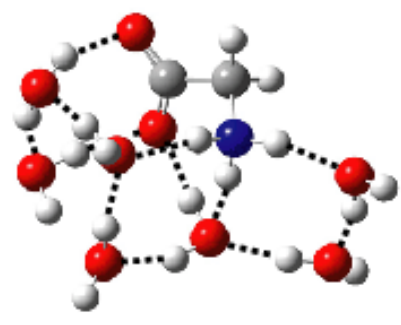

Z7-bb

6.30

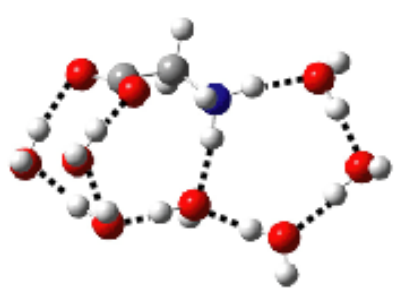

Z7-cc

6.31

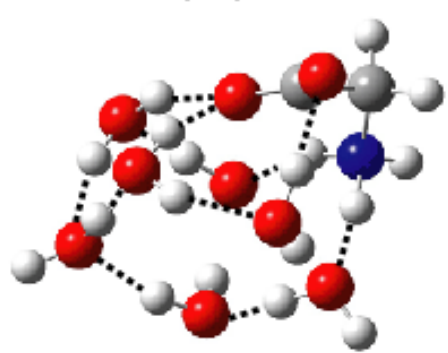

Z7-dd

8.32 